\title{
COUNTING PARTITIONS ON THE ABACUS
}

\author{
MARK WILDON
}

\begin{abstract}
In 2003, Maróti showed that one could use the machinery of $\ell$-cores and $\ell$-quotients of partitions to establish lower bounds for $p(n)$, the number of partitions of $n$. In this paper we explore these ideas in the case $\ell=2$, using them to give a largely combinatorial proof of an effective upper bound on $p(n)$, and to prove asymptotic formulae for the number of self-conjugate partitions, and the number of partitions with distinct parts. In a further application we give a combinatorial proof of an identity originally due to Gauss.
\end{abstract}

\section{INTRODUCTION}

A partition of a number $n \in \mathbf{N}_{0}$ is a sequence $\left(\lambda_{1}, \ldots, \lambda_{k}\right)$ such that $\lambda_{1} \geq \lambda_{2} \geq \ldots \geq \lambda_{k} \geq 1$ and $\lambda_{1}+\ldots+\lambda_{k}=n$. To indicate that $\lambda$ is a partition of $n$ we write $|\lambda|=n$. Let $p(n)$ be the number of partitions of $n$. By our definition, $\emptyset$ is the unique partition of 0 .

The problem of finding asymptotic estimates for $p(n)$ was introduced, and one might reasonably say, solved, by the famous paper of HardyRamanujan [1. As well as their exact formula for $p(n)$, proved as the original application of the circle-method, their paper also contains several weaker results proved using less sophisticated analytic methods; for example, that

$$
\log p(n) \sim c \sqrt{n} \text { as } n \rightarrow \infty
$$

where $c=2 \sqrt{\pi^{2} / 6}$. The only time they refer to using combinatorial arguments to obtain lower and upper bounds for $p(n)$ is in a footnote in $\S 2.1$, which suggests the strongest such bounds they could prove were of the form

$$
2^{A \sqrt{n}}<p(n)<n^{B \sqrt{n}} .
$$

Our first aim is this paper is to improve on this. In $\S 2$ we introduce G. D. James' abacus notation for partitions and use it to establish an important bijection on partitions (see $\S 2.2$ ). In $\S 3$ we use this bijection to show that for every $\varepsilon>0$ there exists a constant $A(\varepsilon)$ such that

$$
\log p(n) \leq A(\varepsilon) n^{1 / 2+\varepsilon} \quad \text { for all } n \geq 0 .
$$

Despite the appearance of an ' $\varepsilon$ ' in this inequality, the proof of (1) is almost entirely combinatorial. The closest competing result in the literature is due to Erdős, who gives at the start of [2] a short elementary proof that

$$
\log p(n) \leq c n^{1 / 2} \text { for all } n \geq 0 \text {, }
$$

where, as before, $c=2 \sqrt{\pi^{2} / 6}$. Erdös' result is of course significantly sharper than (11), but this improvement comes at the cost of a greater contribution

Date: August 3, 2006.

2000 Mathematics Subject Classification. 05A17. 
from analysis. For instance, the reader may already have correctly guessed that the identity $\sum_{n=1}^{\infty} 1 / n^{2}=\pi^{2} / 6$ is needed.

In this context, we mention that Peter Neumann (Oxford Algebra Kinderseminar, January 2003) has asked whether there is a combinatorial proof that there exists a constant $C$ such that $\log p(n) \leq C \sqrt{n}$ for all $n \geq 0$. While (11) comes tantalisingly close, the ideas behind it do not appear quite powerful enough to solve his problem.

Our second aim is to show that the partition bijection given in $\S 2.2$ may be used to solve several other problems in the enumeration of partitions. In our first application we give a very short proof of an identity originally due to Gauss (see [3, end of $\S 3]$ ):

$$
\frac{\left(1-x^{2}\right)\left(1-x^{4}\right)\left(1-x^{6}\right) \ldots}{(1-x)\left(1-x^{3}\right)\left(1-x^{5}\right) \ldots}=\sum_{m \geq 0} x^{\frac{m(m+1)}{2}} .
$$

Our main application concerns a result of Erdős. In the main part of [2], Erdös uses the same underlying idea as he used to prove (2), and some more lengthy (but still elementary) analysis, to prove that there exists a constant $b \in \mathbf{R}$ such that

$$
p(n) \sim \frac{\mathrm{e}^{c \sqrt{n}}}{b n} \text { as } n \rightarrow \infty .
$$

It was already known from the work of Hardy and Ramanujan [1] that $b=4 \sqrt{3}$, but Erdös' methods were not sufficiently powerful to prove this. Later however, Newman [4] gave an elementary proof that if (44) holds for some constant $b$, then necessarily $b=4 \sqrt{3}$. In $\S 4$ we use our bijection to give an alternative proof of this result. In length it is comparable with Newman's, but it requires considerably less knowledge of analysis, and the motivation is perhaps more apparent.

In $\S 5$ we conclude by showing how the abacus may be applied to give exact asymptotic estimates for two other classes of partitions, namely selfconjugate partitions, and partitions with distinct parts.

\section{A partition BIJECTION}

Let $\lambda$ be a partition of $n$. A 2-hook in $\lambda$ consists of two adjacent nodes in the Young diagram of $\lambda$ whose removal leaves the diagram of a partition of $n-2$. By repeatedly removing 2-hooks from $\lambda$ one obtains the 2 -core of $\lambda$. For example, the 2-core of $(6,3,3,1)$ is $(2,1)$, obtained after removing the five 2-hooks indicated in the diagram below.

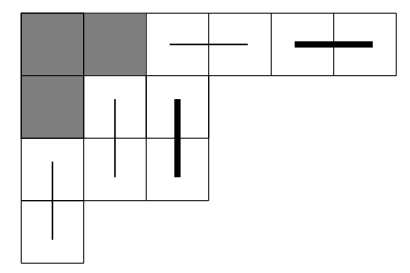

Figure 1 . The 2-core of $(6,3,3,1)$. 
In this diagram, two of the three 2-hooks of the original partition are shown by bold lines. The remaining lines are 2-hooks of partitions obtained en route to the 2 -core.

It is not obvious that the 2-core of a partition is independent of the manner in which one removes its 2-hooks. This, and much else besides, can be seen very clearly if we represent partitions using G. D. James' abacus (see 5, Ch. 2, 26-27]). To make this article self-contained we briefly recall how to operate this piece of apparatus. (We describe only the 'binary' 2-runner abacus.)

2.1. Let $\lambda$ be a partition of $n$. Starting in the southwest corner of the Young diagram of $\lambda$ walk along its rim, heading towards the northeast corner. For each step right, put a space, indicated $\circ$, and for each step up, put a bead, indicated •. For example, the partition $(6,3,3,1)$ shown above has the sequence $\circ \bullet \circ \bullet \bullet \circ \bullet \bullet$. It will be seen shortly that it is useful to allow such a sequence to begin with any number of beads, and to finish with any number of spaces - these must be stripped off before the partition is recovered.

One then arranges the sequence in two columns, known as the runners of the abacus. For instance $(6,3,3,1)$ is represented by

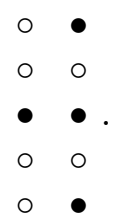

We call this an abacus display for the partition $\lambda$. One sees that 2-hooks in $\lambda$ correspond to beads with a space above them, and that an abacus display for the partition obtained by removing a given 2-hook is obtained by sliding the corresponding bead one space up its runner. (It is at this point that our convention about initial beads and final spaces is needed.) The 2-core of $\lambda$ is obtained by pushing all the beads up as far as they will go.

To reconstruct the partition $\lambda$ from an abacus display for its 2-core we need to know the number of positions down each bead must be moved. For example, given the abacus display

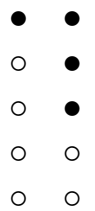

for the 2-core of $(6,3,3,1)$, we recover the original partition by moving the bead on the first runner down by 2 positions, and on the second runner moving the lowest bead down by 2 positions and the next lowest down by 1 position. We record this information in a pair of partitions, $(\mu, \nu)$, known as the 2-quotient of $\lambda$; here $(6,3,1,1)$ has 2 -quotient $((2),(2,1))$. Note that the total number of nodes in $\mu$ and $\nu$ is equal to the total number of 2-hooks removed, so $|\lambda|=|\gamma|+2(|\mu|+|\nu|)$ where $\gamma$ is the 2-core of $\lambda$. 
2.2. Given a partition $\lambda$ we may, by adding a bead at the start if necessary, choose an abacus display for $\lambda$ so that the 2-core of $\lambda$ has at least as many beads on the second runner as the first. With this convention to fix the order of the partitions in the 2-quotient of $\lambda$, the correspondence just described between partitions and pairs $(\gamma,(\mu, \nu))$ of 2-cores and 2-quotients is bijective. Moreover, it is easy to see that any 2 -core is of the form $(m, m-1, \ldots, 1)$ for some $m \in \mathbf{N}_{0}$. Therefore we have shown that, if $t(n)$ is the number of pairs $(\mu, \nu)$ where $\mu$ and $\nu$ are partitions with $|\mu|+|\nu|=n$, then

$$
p(n)=\sum_{r} t\left(\frac{n-r(r+1) / 2}{2}\right)
$$

where the sum is over all the non-negative integers $r$ such that $n-r(r+1) / 2$ is a non-negative even integer. For example,

$$
p(10)=t(5)+t(2)+t(0) .
$$

As it is easy to calculate $t(n)$ given $p(m)$ for $m \leq n$ using the formula $t(n)=\sum_{m=0}^{n} p(m) p(n-m)$, (15) gives us a recurrence relation for the values of the partition function. We exploit this recurrence in $\S 3$ and $\S 4$ below.

2.3. We now prove Gauss' identity. Let $P(x)$ be the ordinary generating function for $p$,

$$
P(x)=p(0)+p(1) x+p(2) x^{2}+\ldots=\prod_{n=1}^{\infty} \frac{1}{1-x^{n}} .
$$

Since each node in the 2-quotient of a partition contributes two nodes to the original partition, our bijection shows that

$$
P(x)=P\left(x^{2}\right)^{2} \sum_{m \geq 0} x^{\frac{m(m+1)}{2}} .
$$

By (6), this is equivalent to Gauss' identity (3).

It should be said that this is not the first combinatorial proof of Gauss' identity. In [6], Andrews gives a bijective proof (in the style of Franklin's proof of Euler's Pentagonal Number Theorem) that:

$$
\frac{\left(1-q^{2}\right)\left(1-q^{4}\right)\left(1-q^{6}\right) \ldots}{(1+q)\left(1+q^{3}\right)\left(1+q^{5}\right) \ldots}=1+\sum_{n=1}^{\infty}(-1)^{n} q^{n(2 n-1)}\left(1+q^{2 n}\right) .
$$

Replacing $q$ with $-x$ gives (3). Another strategy is to first give a combinatorial proof of Jacobi's triple product identity, as done for example in [7, §6.2]; from Jacobi's identity, it only takes a simple specialisation to get (3).

2.4. We conclude this section by remarking that the bijection we have described may be thought as being between the set of all partitions, and the set of all oriented binary trees with vertices labelled by members of $\mathbf{N}_{0}$, with the property that if two leaves are connected to the same node, they are not both labelled by 0 . For example, the 2 -core and 2 -quotient of $(6,3,3,1)$ may be represented by the diagram below.

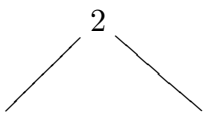


Here the upper ' 2 ' records the number of rows (or columns) in the 2-core of $(6,3,3,1)$. Iterating the process, we find that $(6,3,3,1)$ is represented by the oriented binary tree shown below.

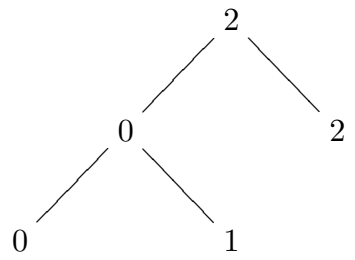

The corresponding formulation of Gauss' identity is the attractive

$$
\prod_{n=1}^{\infty} \frac{1}{1-x^{n}}=\prod_{m=0}^{\infty}\left(\sum_{r=0}^{\infty} x^{\frac{r(r+1)}{2} 2^{m}}\right)^{2^{m}} .
$$

\section{LOWER AND UPPER BOUNDS FOR $p(n)$}

By counting only partitions of $4 m$ with 2-core $\emptyset$ and 2-quotient $(\mu, \nu)$ where both $\mu$ and $\nu$ are partitions of $m$, we obtain the inequality $p(4 m) \geq$ $p(m)^{2}$. An immediate consequence is that

$$
p\left(4^{r}\right) \geq p(4)^{2^{r-1}}=5^{2^{r-1}} \quad \text { for all } r \geq 1 .
$$

Given $n \in \mathbf{N}$, we may choose a power of 4 , say $4^{r}$, such that $n / 4<4^{r} \leq n$. Since $p$ is an increasing function, $p(n) \geq p\left(4^{r}\right)$, and so

$$
\log p(n) \geq \log p\left(4^{r}\right) \geq \frac{\log 5}{2} 2^{r} \geq \frac{\log 5}{4} \sqrt{n} \text { for all } n \geq 4 .
$$

It seems striking that such a simple argument gives a lower bound which, while far from optimal, is still of the right asymptotic form.

Maróti (see [8, Corollary 3.1]) has shown that, with a little more analysis, and the use of a computer to check small cases, one can obtain the significantly stronger lower bound

$$
p(n) \geq \frac{\mathrm{e}^{2 \sqrt{n}}}{14} \quad \text { for all } n \geq 1 .
$$

The next lemma shows how our bijection may be applied to give an upper bound.

Lemma 3.1. Suppose that $f: \mathbf{R}_{\geq 0} \rightarrow \mathbf{R}_{\geq 0}$ is an increasing function such that $f(0) \geq 1$ and

$$
\sum_{0 \leq s \leq n / 2} f\left(\frac{n}{2}-s\right) f(s) \leq \frac{f(n)}{n} \quad \text { for all } n \in \mathbf{N} .
$$

Then $p(n) \leq f(n)$ for all $n \geq 1$.

Proof. We work by induction on $n$. Using (5) we have

$$
p(n) \leq n t(n / 2)=n \sum_{0 \leq s \leq n / 2} p\left(\frac{n}{2}-s\right) p(s)
$$


where we adopt the convention that $p(x)=p(\lfloor x\rfloor)$, and similarly for $t(x)$. Hence

$$
p(n) \leq n \sum_{0 \leq s \leq n / 2} f\left(\frac{n}{2}-s\right) f(s) \leq f(n)
$$

which completes the inductive step.

The reader will see that we have been very crude in taking $n$ as an upper bound for the number of summands in (5) - there appears to be no advantage in being any more precise at this point.

Theorem 3.2. Given any $\varepsilon>0$ there is a constant $A(\varepsilon)$ depending only on $\varepsilon$ such that

$$
p(n) \leq \mathrm{e}^{A(\varepsilon) n^{\frac{1}{2}+\varepsilon}} \quad \text { for all } n \geq 0 .
$$

Proof. Without loss of generality, assume that $\varepsilon<\frac{1}{2}$. Let $f(x)=\mathrm{e}^{A x^{\frac{1}{2}+\varepsilon}}$. We shall prove that when $A$ is large enough, $f$ satisfies the conditions of Lemma 3.1 Setting $\beta=\frac{1}{2}+\varepsilon$, we have

$$
\sum_{0 \leq s \leq n / 2} f(n / 2-s) f(s)=\sum_{0 \leq s \leq n / 2} \mathrm{e}^{A(n / 2-s)^{\beta}+A s^{\beta}} \leq \frac{n+1}{2} \mathrm{e}^{A n^{\beta} 4^{-\varepsilon}}
$$

where we used the inequality

$$
x^{\beta}+y^{\beta} \leq 2\left(\frac{x+y}{2}\right)^{\beta}
$$

which is valid for $0 \leq \beta \leq 1$ and $x, y \geq 0$. Therefore the only condition we must satisfy is that

$$
\mathrm{e}^{A n^{\beta} 4^{-\varepsilon}} \leq \frac{2}{n(n+1)} \mathrm{e}^{A n^{\beta}} \quad \text { for all } n \geq 1,
$$

or, equivalently, that

$$
\mathrm{e}^{A\left(1-4^{-\varepsilon}\right) n^{\beta}} \geq \frac{n(n+1)}{2} \text { for all } n \geq 1 .
$$

Clearly this will hold provided $A$ is sufficient large.

\section{A new eVAluation of the CONSTANT in ERdő' FORMUla}

In this section we assume Erdös' result (4) that $p(n) \sim \mathrm{e}^{c \sqrt{n}} / b n$ for some constant $b \in \mathbf{R}$, and prove that $b=4 \sqrt{3}$. We shall also need Erdös' upper bound (2) that $p(n) \leq \mathrm{e}^{c \sqrt{n}}$ for all $n \geq 1$. (Here, as always, $c=2 \sqrt{\pi^{2} / 6}$.)

Recall that $t(n)$ denotes the number of pairs $(\mu, \nu)$ of partitions such that $|\mu|+|\nu|=n$. We first show that it follows from Erdös' result that

$$
t(n) \sim \frac{\mathrm{e}^{c \sqrt{2 n}}}{n^{5 / 4}} \frac{2^{2} 3^{1 / 4}}{b^{2}} \text { as } n \rightarrow \infty .
$$

We then use the bijection described in $\S 2.2$ to deduce that

$$
p(32 m) \sim \frac{\mathrm{e}^{c \sqrt{32 m}}}{32 m} \frac{2^{2} 3^{1 / 2}}{b^{2}} \text { as } n \rightarrow \infty .
$$

Comparing this with (4) shows that $b=4 \sqrt{3}$. (The only reason for taking $32 m$ rather than a general $n$ in (8) is that this choice lead to some 
simplifications in the expressions we encounter; there is of course no need to prove anything stronger.)

4.1. All the results in this section and $\S 5$ are proved using the following simple lemma, whose proof requires only the basic integral

$$
\int_{0}^{\infty} \mathrm{e}^{-\gamma x^{2}} \mathrm{~d} x=\sqrt{\frac{\pi}{4 \gamma}} \text { if } \gamma>0
$$

Lemma 4.1. If $\alpha, \beta, \gamma, \vartheta>0$ then

$$
\sum_{r=0}^{\alpha m^{\beta+\vartheta}} \mathrm{e}^{-\gamma r^{2} / m^{2 \beta}} \sim \sqrt{\frac{\pi}{4 \gamma}} m^{\beta} \quad \text { as } m \rightarrow \infty .
$$

Proof. Let $S_{m}$ stand for the sum on the left-hand-side. Since

$$
S_{m}-1 \leq \int_{0}^{\alpha m^{\beta+\vartheta}} \mathrm{e}^{-\gamma x^{2} / m^{2 \beta}} \mathrm{d} x \leq S_{m},
$$

it is sufficient to prove that

$$
J_{m}=\frac{1}{m^{\beta}} \int_{0}^{\alpha m^{\beta+\vartheta}} \mathrm{e}^{-\gamma x^{2} / m^{2 \beta}} \mathrm{d} x \rightarrow \sqrt{\frac{\pi}{4 \gamma}} \text { as } m \rightarrow \infty .
$$

This is not hard. On the one hand,

$$
J_{m} \leq \frac{1}{m^{\beta}} \int_{0}^{\infty} \mathrm{e}^{-\gamma x^{2} / m^{2 \beta}} \mathrm{d} x=\sqrt{\frac{\pi}{4 \gamma}}
$$

and on the other,

$\sqrt{\frac{\pi}{4 \gamma}}-J_{m}=\frac{1}{m^{\beta}} \int_{\alpha m^{\beta+\vartheta}}^{\infty} \mathrm{e}^{-\gamma x^{2} / m^{2 \beta}} \mathrm{d} x=\frac{1}{m^{\beta}} \int_{\alpha m^{\vartheta}}^{\infty} \mathrm{e}^{-\gamma y^{2}} m^{\beta} \mathrm{d} y=\int_{\alpha m^{\vartheta}}^{\infty} \mathrm{e}^{-\gamma y^{2}} \mathrm{~d} y$ which tends to 0 as $m \rightarrow \infty$. Hence $J_{m}$ has the claimed limit.

4.2. We start by proving (7). If (7) holds when $n$ is even then, by a simple sandwiching argument, it must hold for all $n$. We may therefore assume that $n=2 m$, in which case (7) becomes

$$
t(2 m) \sim \frac{\mathrm{e}^{2 c \sqrt{m}}}{m^{5 / 4}} \frac{2^{3 / 4} 3^{1 / 4}}{b^{2}} \text { as } n \rightarrow \infty .
$$

Let $\varepsilon>0$ be given. Choose $N$ so that

$$
1-\varepsilon<\frac{p(n)}{e^{c \sqrt{n}} / b n}<1+\varepsilon \text { for all } n \geq N .
$$

We first obtain an upper estimate for $t(2 m)$. For $0<\alpha<1$ set

$$
\begin{aligned}
M_{\alpha}(m) & =\sum_{0 \leq r \leq \alpha m} p(m+r) p(m-r), \\
R_{\alpha}(m) & =\sum_{\alpha m<r \leq m} p(m+r) p(m-r) .
\end{aligned}
$$

so we have $t(2 m) / 2 \leq M_{\alpha}(m)+R_{\alpha}(m)$. We shall see that as $m$ tends to infinity, the contribution of $M_{\alpha}(m)$ to $t(2 m)$ dominates, no matter what the choice of $\alpha$. In fact, it follows from Erdős' bound (2) and the inequality

$$
\sqrt{1+x}+\sqrt{1-x} \leq 2-\frac{x^{2}}{4} \quad \text { if } 0 \leq x \leq 1
$$


that

$$
R_{\alpha}(m) \leq \sum_{\alpha m<r \leq m} \mathrm{e}^{c \sqrt{m}(\sqrt{1+r / m}+\sqrt{1-r / m})} \leq(1-\alpha) m e^{2 c \sqrt{m}-c r^{2} / 4 m^{3 / 2}},
$$

and so

$$
\frac{R_{\alpha}(m)}{\mathrm{e}^{2 c \sqrt{m}} / m^{5 / 4}} \leq(1-\alpha) m^{9 / 4} \mathrm{e}^{-c \alpha^{2} \sqrt{m} / 4} \rightarrow 0 \quad \text { as } m \rightarrow \infty .
$$

We now look at the main contribution. Provided $\alpha<1 / 2$ and $m \geq 2 N$, the smallest $n$ for which $p(n)$ appears in $M_{\alpha}(m)$ is at least $N$. Hence if these conditions hold,

$$
\begin{aligned}
\frac{M_{\alpha}(m)}{(1+\varepsilon)^{2}} & \leq \sum_{r=0}^{\alpha m} \frac{\mathrm{e}^{c \sqrt{m}(\sqrt{1+r / m}+\sqrt{1-r / m})}}{b^{2}\left(m^{2}-r^{2}\right)} \\
& \leq \frac{\mathrm{e}^{2 c \sqrt{m}}}{b^{2} m^{2}\left(1-\alpha^{2}\right)} \sum_{r=0}^{\alpha m} \mathrm{e}^{c \sqrt{m}(\sqrt{1+r / m}+\sqrt{1-r / m}-2)} \\
& \leq \frac{\mathrm{e}^{2 c \sqrt{m}}}{b^{2} m^{2}\left(1-\alpha^{2}\right)} \sum_{r=0}^{\alpha m} \mathrm{e}^{-c r^{2} / 4 m^{3 / 2}} .
\end{aligned}
$$

It now follows from Lemma 4.1, applied with $\beta=3 / 4, \vartheta=1 / 4$, that

$$
\limsup \frac{M_{\alpha}(m)}{\mathrm{e}^{2 c \sqrt{m}} / m^{5 / 4}} \leq \frac{(1+\varepsilon)^{2}}{b^{2}\left(1-\alpha^{2}\right)} \sqrt{\frac{\pi}{c}}=\frac{(1+\varepsilon)^{2} 3^{1 / 4}}{2^{1 / 4} b^{2}\left(1-\alpha^{2}\right)} .
$$

Finally, let $\alpha, \varepsilon \rightarrow 0$ to get

$$
\lim \sup \frac{t(2 m)}{\mathrm{e}^{2 c \sqrt{m}} / m^{5 / 4}} \leq \frac{2^{3 / 4} 3^{1 / 4}}{b^{2}} .
$$

We now obtain a lower estimate for $t(2 m)$. By taking $\alpha$ sufficiently small we may ensure that

$$
\sqrt{1+x}+\sqrt{1-x} \geq 2-\frac{(1+\varepsilon) x^{2}}{4} \text { if } 0 \leq x<\alpha .
$$

For this choice of $\alpha$ we have, by the same manipulations as before,

$$
\frac{t(2 m)}{e^{2 c \sqrt{m}} / m^{5 / 4}}+\frac{p(m)^{2}}{e^{2 c \sqrt{m}} / m^{5 / 4}} \geq \frac{2}{b^{2} m^{3 / 4}} \sum_{r=0}^{\alpha m} \mathrm{e}^{-c r^{2}(1+\varepsilon) / 4 m^{3 / 2}} .
$$

The second term on the left-hand-side compensates for the double counting of the contribution of $p(m) p(m)$ to $t(2 m)$; as $p(m) \leq(1+\varepsilon) \mathrm{e}^{c \sqrt{m}} / b m$ for $m \geq N$, this term vanishes in the limit. As before, it follows from Lemma 4.1 that

$$
\liminf \frac{t(2 m)}{e^{2 c \sqrt{m}} / m^{5 / 4}} \geq \frac{1}{b^{2}} \sqrt{\frac{\pi}{c(1+\varepsilon)}}=\frac{2^{3 / 4} 3^{1 / 4}}{b^{2} \sqrt{1+\varepsilon}} .
$$

Now let $\varepsilon \rightarrow 0$. This completes the proof of (9). 
4.3. It follows from (5) that

$$
p(32 m)=\sum_{r} t\left(16 m-\frac{r(r+1)}{4}\right)
$$

where the sum is over all non-negative integers $r$ such that $16 m-r(r+1) / 4$ is a non-negative even number. We may estimate this sum using the same ideas as $\S 4.2$.

We start with a lower estimate. The contribution from those $r \equiv 0 \bmod 4$ is at least the contribution from those $r \equiv 3 \bmod 4$, so we have

$$
p(32 m) \geq 2 \sum_{s=1}^{\alpha \sqrt{m}} t(16 m-(4 s-1) s)
$$

for any $\alpha$ such that $0<\alpha<1$.

Let $\varepsilon>0$ be given. By (7) we may choose $N$ so that

$$
t(n)>(1-\varepsilon) \frac{\mathrm{e}^{c \sqrt{2 n}}}{n^{5 / 4}} \frac{2^{2} 3^{1 / 4}}{b^{2}} \text { for all } n \geq N .
$$

Take $m \geq N$. Choose $\alpha$ sufficiently small that

$$
\sqrt{1-x} \geq 1-\frac{(1+\varepsilon) x}{2} \quad \text { if } 0 \leq x \leq \frac{\alpha^{2}}{4} .
$$

For this choice of $\alpha$ we have

$$
\begin{aligned}
\frac{p(32 m)}{1-\varepsilon} & \geq 2 \sum_{s=1}^{\alpha \sqrt{m}} \frac{2^{2} 3^{1 / 4}}{b^{2}} \frac{\mathrm{e}^{c \sqrt{32 m-2 s(4 s-1)}}}{(16 m-s(4 s-1))^{5 / 4}} \\
& \geq \frac{2^{3} 3^{1 / 4}}{b^{2}} \sum_{s=1}^{\alpha \sqrt{m}} \frac{\mathrm{e}^{c \sqrt{32 m} \sqrt{1-\frac{s^{2}}{4 m}}}}{(16 m-s(4 s-1))^{5 / 4}} \\
& \geq \frac{\mathrm{e}^{c \sqrt{32 m}}}{32 m} \frac{2^{3} 3^{1 / 4}}{b^{2} m^{5 / 4}} \sum_{s=1}^{\alpha \sqrt{m}} \mathrm{e}^{-(1+\varepsilon) c s^{2} / \sqrt{2 m}} .
\end{aligned}
$$

It now follows from Lemma 4.1, applied with $\beta=1 / 4, \vartheta=1 / 4$, that

$$
\liminf \frac{p(32 m)}{\mathrm{e}^{c \sqrt{32 m}} / 32 m} \geq \frac{(1-\varepsilon)}{\sqrt{1+\varepsilon}} \frac{2^{3} 3^{1 / 4}}{b^{2}} \sqrt{\frac{\pi \sqrt{2}}{4 c}}=\frac{(1-\varepsilon)}{\sqrt{1+\varepsilon}} \frac{2^{2} 3^{1 / 2}}{b^{2}} .
$$

Now let $\varepsilon \rightarrow 0$.

To complete the proof of (8) we must also show that

$$
\limsup \frac{p(32 m)}{\mathrm{e}^{c \sqrt{32 m}} / 32 m} \leq \frac{2^{2} 3^{1 / 2}}{b^{2}} .
$$

In order to apply the method used in $\S 4.2$ we need a uniform upper bound for $t(n)$; the relatively crude estimate

$$
t(n) \leq n p(n / 2)^{2} \leq n \mathrm{e}^{c \sqrt{2 n}} \text { for } n \geq 1
$$

given by (2) is sufficient for this purpose. As no other new ideas are needed we shall omit the remaining details of the proof. 


\section{Asymptotic Formulae For two SPECIAL types OF PARTition}

In this final section we give asymptotic formulae for the number of selfconjugate partitions, and for the number of partitions with distinct parts. As corollaries, we get precise estimates for the proportions of such partitions. We omit the details of the analytic arguments required, as they are of the same nature as those already seen in $\S 4$.

5.1. Self-conjugate partitions. Recall that if $\lambda=\left(\lambda_{1}, \ldots, \lambda_{k}\right)$ is a partition of $n$ then the conjugate partition $\lambda^{\prime}$ is defined by $\lambda_{j}^{\prime}=\left|\left\{i: \lambda_{i} \geq j\right\}\right|$; the diagram of $\lambda^{\prime}$ is obtained from that of $\lambda$ by reflecting it in its leading diagonal. Let $s(n)$ be the number of self-conjugate partitions of $n$.

Given an abacus display for a partition $\lambda$, one obtains a display for the conjugate partition $\lambda^{\prime}$ by reading the sequence of beads and spaces from right to left, and then replacing • with $\circ$ and vice versa. For example, $(6,3,3,1)$ and $(6,3,3,1)^{\prime}=(4,3,3,1,1,1)$ have the abacus displays:

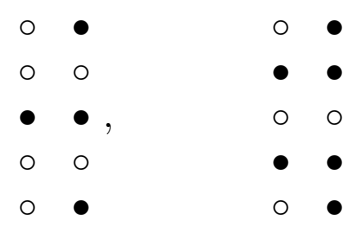

respectively.

If $\lambda$ is self-conjugate then the sequence of spaces and beads corresponding to $\lambda$ has an even number of entries. It follows that if $\lambda$ has 2-quotient $(\mu, \nu)$ then $\nu=\mu^{\prime}$. This gives us a bijection between self-conjugate partitions of $n$ and pairs $(\gamma, \mu)$ where $\gamma$ is a 2-core (and so of a known shape) and $\mu$ is a partition of $(n-|\gamma|) / 4$. (This bijection is well known, see for instance [5, Theorem 6.2.2]. We leave it to the reader to work out the connection with the labelled binary trees of $\S 2.4$.)

The corresponding numerical result is

$$
s(n)=\sum p\left(\frac{n-r(r+1) / 2}{4}\right)
$$

where the sum is over all the non-negative integers $r$ such that $n-r(r+1) / 2$ is a non-negative integer divisible by 4 . By the same techniques used in $\S 4.3$, one finds that

$$
s(n) \sim \frac{\mathrm{e}^{\frac{c}{2} \sqrt{n}}}{2^{7 / 4} 3^{1 / 4} n^{3 / 4}} \quad \text { as } n \rightarrow \infty .
$$

It follows that the proportion of self-conjugate partitions satisfies

$$
\frac{s(n)}{p(n)} \sim(6 n)^{1 / 4} \mathrm{e}^{-\frac{c \sqrt{n}}{2}} \quad \text { as } n \rightarrow \infty
$$

Thus of the $p(n)$ partitions of $n$, approximately $\sqrt{p(n)}$ are self-conjugate.

5.2. Partitions with distinct parts. Let $q(n)$ be the number of partitions of $n$ with distinct parts. Here the numerical result we require is

$$
q(n)=\sum p\left(\frac{n-r(r+1) / 2}{2}\right)
$$


where the sum is over all the non-negative integers $r$ such that $n-r(r+1) / 2$ is a non-negative even integer. For a combinatorial proof of this result, see Proposition 5.2 in $[9$. As an alternative, note that the generating function for $q(n)$ is

$$
Q(x)=\prod_{n=1}^{\infty}\left(1+x^{n}\right)=\frac{P(x)}{P\left(x^{2}\right)} .
$$

Hence by (3),

$$
Q(x)=P\left(x^{2}\right) \sum_{m \geq 0} x^{\frac{m(m+1)}{2}} .
$$

Comparing coefficients of $x^{n}$ gives the required result.

By the same techniques used in $\S 4.3$, one finds that

$$
q(n) \sim \frac{\mathrm{e}^{\frac{c}{\sqrt{2}} \sqrt{n}}}{2^{2} 3^{1 / 4} n^{3 / 4}} \quad \text { as } n \rightarrow \infty .
$$

It follows that the proportion of partitions with distinct parts satisfies

$$
\frac{q(n)}{p(n)} \sim(3 n)^{1 / 4} \mathrm{e}^{-c \sqrt{n}\left(1-\frac{1}{\sqrt{2}}\right)} \quad \text { as } n \rightarrow \infty .
$$

\section{AcKnowledgements}

I should like to thank John Britnell and Atilla Maróti for several helpful conversations on the subject of partitions, and Gordon James and Peter Neumann for asking two of the questions which motivated this paper.

\section{REFERENCES}

[1] G. H. Hardy and S. Ramanujan, "Asymptotic formulae in combinatorial analysis", Proc. London Math. Soc. (2), vol. 17, pp. 75-113, 1917.

[2] P. Erdös, "On an elementary proof of some asymptotic formulas in the theory of partitions", Ann. of Math. (2), vol. 43, pp. 437-450, 1942.

[3] C. F. Gauss, "Zur Theorie der Transcendenten Functionen Gehörig", in Werke, vol. III, pp. 435-446. Königslichen Gesellschaft der Wissenschaften zu Göttingen, 1886.

[4] D. J. Newman, "The evaluation of the constant in the formula for the number of partitions of n", Amer. J. Math., vol. 73, pp. 599-601, 1951.

[5] G. James and A. Kerber, The representation theory of the symmetric group, vol. 16 of Encyclopedia of Mathematics and its Applications, Addison-Wesley Publishing Co., Reading, Mass., 1981.

[6] G. E. Andrews, "Two theorems of Gauss and allied identities proved arithmetically", Pacific J. Math., vol. 41, pp. 563-578, 1972.

[7] I. Pak, "Partition bijections, a survey", To appear in Ramanujan J., 69 pp.

[8] A. Maróti, "On elementary lower bounds for the partition function", Integers, vol. 3, pp. A10, 9 pp. (electronic), 2003.

[9] B. Külshammer, J. B. Olsson, and G. R. Robinson, "Generalized blocks for symmetric groups", Invent. Math., vol. 151, no. 3, pp. 513-552, 2003.

E-mail address: M.J.Wildon@swansea.ac.uk. 